\title{
Reimagining the Future of HIV Service Implementation in the Philippines Based on Lessons from COVID-19
}

\author{
Ma. Irene N. Quilantang ${ }^{1,2} \cdot$ Amiel Nazer C. Bermudez $^{1,3} \cdot$ Don Operario $^{1}$
}

Published online: 25 May 2020

○) Springer Science+Business Media, LLC, part of Springer Nature 2020

The Philippines has been characterized as having one of the fastest growing HIV epidemics in the world, following from the rapid and continued rise in new diagnoses since 2007 [1]. Community-based organizations (CBOs) play an important role in the Philippine government's efforts to mitigate the HIV epidemic. Confronted with multiple challenges imposed by the COVID-19 epidemic, HIV-focused CBOs have implemented new practices to ensure continuous provision of treatment, care, and support services for persons living with HIV (PLHIV). In light of the country's nascent and evolving HIV policies, newly developed strategies in the delivery of HIV services can provide a blueprint for reimagining HIV program implementation in the Philippines, both during and beyond the COVID-19 crisis.

\section{Adaptations in HIV Service Delivery Due to COVID-19}

The COVID-19 epidemic emerged in the Philippines during a crucial time in the country's response to the HIV epidemic. National capacity to test, treat, and prevent further infections was initially challenged by limitations in healthcare infrastructure, human resources to provide HIV-related services, and lack of local HIV research to guide country-specific policies [2]. The government passed the updated Philippine HIV and AIDS Policy Act (Republic Act 11166) in 2019, committing to a multi-sectoral approach to ensure access to prevention and testing programs as well as investment

Ma. Irene N. Quilantang

irene_quilantang1@brown.edu

1 Brown University School of Public Health, Providence, Rhode Island, USA

2 College of Arts and Sciences, University of the Philippines Manila, Manila, Philippines

3 College of Public Health, University of the Philippines Manila, Manila, Philippines in treatment, care, and support services for persons living with HIV [3]. However, unforeseen challenges emerging in the context of the COVID-19 epidemic reveal areas for considering long-term strategies to HIV service delivery by Philippines-based CBOs in future crisis conditions.

The implementation of community quarantine measures due to COVID-19 in most parts of the Philippines has restricted transportation services to individuals performing essential functions. As such, CBO staff and volunteers who perform HIV screening, counseling, and referrals must work remotely, thereby restricting the number of clients they can serve. This limited capacity for HIV screening, and pre- and post-test counselling provided by $\mathrm{CBO}$ can lead to delays in HIV diagnosis and further delays in the initiation of treatment. In response to the need for providing ongoing support during the COVID-19 crisis, some CBOs have designated "skeletal workforces" comprising a subset of core staff/volunteers who continue to deliver basic services to PLHIV and members of high-risk groups, even during the most acute phases of community quarantine implementation and physical distancing measures. For example, skeletal workforces can ensure that PLHIV receive critical counseling and treatment referrals, and can provide community-based HIV screening (e.g. using rapid HIV test kits) and linkage to confirmatory testing. Designation of skeletal workforces at HIV-focused CBOs will be an adaptive strategy beyond the COVID-19 crisis, in order to assure provision of basic HIV services during future emergency conditions that compromise CBOs' full organizational capacities.

Face-to-face consultations between PLHIV and providers have decreased or ceased due to travel restrictions and physical distancing advisories. These changes have serious consequences for PLHIV who must address health problems on their own or with minimal support, without the benefit of expert advice. Some CBOs have responded to this challenge by creating and maintaining online platforms, and adapting telemedicine for clinical consultations and counseling. However, an arising concern relates to confidentiality of patient 
information, especially if data privacy safeguards are not observed. In addition, access to telemedicine platforms is premised on quality access to telecommunication services (e.g. decent internet connection, mobile phone data). Thus, economically-disadvantaged PLHIV and those in rural locations may have limited access to these technologies. These emerging issues must be addressed by Philippines-based CBOs if telemedicine or remote counseling strategies are used in future service delivery.

Travel restrictions and physical distancing measures in the Philippines have also disrupted the supply and distribution chain for essential ARV drugs, which may decrease adherence to treatment. The Philippine Department of Health (DOH) has recommended that ART treatment centers and primary HIV facilities adopt innovative and assertive medication delivery strategies to ensure access to treatment, while also minimizing risk for patient and staff exposure to COVID-19 [4]. In response, CBOs have started delivering ART medications to PLHIV at designated drop-off points. These "courier" services are initiated through online requests submitted by PLHIV, which detail the location and preferred time/date of delivery. This service has been aided by the development of mobile applications that include a map of ARV medication distribution points. However, access to these technologies may be limited for economically and technologically disadvantaged PLHIV. In addition, access to drop-off points remains challenging in geographic settings outside of main metropolitan areas such as Metro Manila and Metro Cebu. The topography of the Philippines complicates the scalability of this innovative medication delivery strategy (e.g., due to the country's mountainous terrain, archipelago dispersion, and limited roads system in rural settings). Sustainability of "courier" services beyond the COVID-19 crisis depends on CBOs' human, financial, and material resources to provide app-based medication delivery. Given the unclear duration of the COVID-19 crisis and the possible diversion of donor support from CBOs to health care facilities treating COVID-19 patients, CBOs may not be able to sustainably finance these initiatives.

\section{A Reimagined Blueprint for the Provision of HIV Treatment, Care and Support Services}

Despite challenges in the provision of HIV treatment, care, and support services arising from the COVID-19 epidemic in the Philippines, CBOs have found ways to continue provision of life-sustaining services. Although these innovative approaches have risen out of epidemic necessity, they are worth considering as key components in the future of the Philippines' HIV policy implementation.

First, online service delivery (e.g., counseling, treatment referrals) should be a viable and acceptable option for HIV-service organizations, both during and beyond the COVID-19 crisis. Expanding ways by which PLHIV can access these services can help unburden the health system by prioritizing facility-based service delivery to more urgent cases. However, these online platforms should be configured in such a way that data privacy is protected. In addition, CBOs and healthcare settings must provide appropriate training, supervision, and support to staff and providers whose roles might shift to telemedicine.

Second, innovative strategies to improve the supply chain of ART medications to PLHIV can arise from the COVID19 crisis. The DOH can consider designating district-level health facilities as ART treatment hubs, in order to more equitably reach PLHIV outside of the country's major metropolitan settings. The adoption of app-based ART delivery and courier services by CBOs is an innovative practice holding promise for further scalability. Further investment and development of public-private partnerships between the government, technology companies, and private courier services are needed to sustain this service.

Third, the DOH should reconsider its policy specifying that PLHIV can only avail their supply of ARV drugs from a designated treatment hub. This requirement is limiting in both COVID-19 and post-COVID-19 contexts, exemplified by recent travel restrictions that have stranded PLHIV in areas far from their designated treatment hub. Recent DOH guidelines in response to the COVID-19 crisis have relaxed this rule, by stipulating that PLHIV affected by the community quarantine can obtain their ART medications from other treatment facilities [4]. Retention of this policy can minimize patients' barriers to ARV medications attributed to their location and geography. Moreover, multi-month ARV drug prescriptions can minimize the need for repeated travel to treatment hubs.

In summary, the COVID-19 crisis provides an opportunity to consider key gaps and weaknesses in HIV service delivery in the Philippines. It is instructive to reflect on innovative practices adopted by CBOs in the context of the COVID-19 crisis that have been made to ensure the continued provision of treatment, care, and support services for PLHIV. Although the immediate, acute challenges imposed by the COVID-19 crisis might abate with time, there will likely emerge other epidemiological, natural, or humanmade crises that restrict healthcare and CBO capacities to provide life-sustaining HIV services. The Philippines must not be caught unprepared for dealing with these eventualities, and must reimagine and invest in more innovative, assertive, and sustainable HIV service delivery strategies.

Acknowledgements Authors were supported by grants from NIH Fogarty International Center (D43TW000237) and NIAID (P30AI042853). The content is solely the responsibility of the authors and does not necessarily represent the official views of the National Institutes of Health. We wish to convey our appreciation to the following community-based 
organizations who provide treatment, care, and support services for persons living with HIV in the Philippines: The Red Whistle, LoveYourself, Inc., Pinoy Plus Advocacy Pilipinas Inc., Positive Action Foundation Philippines, Inc. (PAFPI), MapBeks, and Sustained Health Initiative of the Philippines (SHIP).

\section{Compliance with Ethical Standards}

Conflict of interest Each of the authors declare that they have no conflicts of interest.

\section{References}

Joint United Nations Programme on HIV/AIDS. UNAIDS Report on the Global AIDS Pandemic. 2012. https://www.unaids.org/sites
/default/files/media_asset/20121120_UNAIDS_Global_Repor t_2012_with_annexes_en_1.pdf.

World Health Organization. External review of the national health sector response to HIV and sexually transmitted infections 2013: Republic of the Philippines. Geneva: World Health Organization. 2015. https://iris.wpro.who.int/bitstream/handle/10665.1/11217 19789290617068_eng.pdf.

Congress of the Philippines. Republic Act No. 11166. 2018. https:// www.officialgazette.gov.ph/2018/12/20/republic-act-no-11166/.

Department of Health (Philippines). 2020. Advisory: Access to Antiretroviral (ARV) Drugs of People Living with HIB (PLHIV) Amidst Enhanced Community Quarantine. Philippines.

Publisher's Note Springer Nature remains neutral with regard to jurisdictional claims in published maps and institutional affiliations. 